\title{
Supplementary Information of Distinguishing the Structures of High-Pressure Hydrides with Nuclear Magnetic Resonance Spectroscopy
}

\author{
Da Chen, Wang Gao,* and Qing Jiang*
}

Key Laboratory of Automobile Materials, Ministry of Education, and School of Materials Science and Engineering, Jilin University, Changchun 130022, China.

Corresponding Author*email: wgao@jlu.edu.cn, jiangq@jlu.edu.cn

\section{Computational details}

Since the electric field gradient (EFG) tensor is very sensitive to the geometry, we have used three different methods to optimize the structures in the castep plane-wave code $^{1}$ : semi-local Perdew-Burke-Ernzerhof $(\mathrm{PBE})^{2}$ functional, PBE functional augmented with Tkatchenko and Scheffer $(\mathrm{TS})^{3}$ van der Waals correction (PBE+TS) and hybrid Heyd-Scuseria-Ernzerhof $(\mathrm{HSE})^{4}$ functional. The PBE and PBE+TS calculations use the on-the-fly pseudopotential with the plane-wave energy cutoff of $1000 \mathrm{eV}$ and the Monkhorst-Pack Brillouin ${ }^{5}$ zone sampling grid of spacing $2 \pi \times 0.03$ $\AA^{-1}$. For HSE calculations, we have used the norm-conserving pseudopotentials with the plane-wave energy cutoff of $500 \mathrm{eV}$ and the Monkhorst-Pack Brillouin zone sampling grid of spacing $2 \pi \times 0.05 \AA^{-1}$. For all calculations, the force on atoms is less than $0.001 \mathrm{eV} / \AA ̊$. 
The quadrupolar coupling constant $C_{Q}$ and the quadrupolar asymmetry parameter $\eta_{Q}$ are calculated using PBE functional with the gauge-including projector augmented waves theory ${ }^{6}$ as implemented in castep plane-wave code. The computational parameters are the same as those described above for optimizing the structure with PBE and PBE+TS methods. The nuclear quadrupolar moments are $2.86 \mathrm{mb}$ for ${ }^{2} \mathrm{H},-67.8 \mathrm{mb}$ for ${ }^{33} \mathrm{~S}$ and $200 \mathrm{mb}$ for ${ }^{139} \mathrm{La}$, respectively. ${ }^{7}$ We have tested the dependence of the EFG tensor parameters on the calculation method, showing that the $C_{Q}$ and $\eta_{Q}$ values calculated by PBE+TS method are almost the same as that by PBE method, which is consistent with Monserrat et al.'s results about the independence of the chemical shielding of high-pressure hydrogen on the calculation method. ${ }^{8}$ 


\section{Test of the effect of the optimized methods on the EFG tensor parameters}

Table S1. The EFG tensor parameters of $I m-3 m$ phase for $\mathrm{H}_{3} \mathrm{~S}$ at $150 \mathrm{GPa}$ (the structure was optimized by different methods).

\begin{tabular}{ccccccc}
\hline $\begin{array}{c}\mathrm{H}_{3} \mathrm{~S}-\mathrm{Im}-3 m-150 \\
\mathrm{GPa}\end{array}$ & \multicolumn{3}{c}{$\mid C_{Q}(\mathrm{MHz})$} & \multicolumn{3}{c}{$\eta_{Q}$} \\
\hline $\begin{array}{c}\text { Atomic site } \times \mathrm{N} \\
{ }^{2} \mathrm{H} \times 6\end{array}$ & PBE & PBE+TS & HSE & PBE & PBE+TS & HSE \\
${ }^{33} \mathrm{~S} \times 2$ & $6.01 \mathrm{E}-02$ & $6.38 \mathrm{E}-02$ & $6.32 \mathrm{E}-02$ & 0.00 & 0.00 & 0.00 \\
\hline
\end{tabular}

Table S2. The EFG tensor parameters of $R 3 m$ phase for $\mathrm{H}_{3} \mathrm{~S}$ at $150 \mathrm{GPa}$ (the structure was optimized by different methods).

\begin{tabular}{ccccccc}
\hline $\begin{array}{c}\mathrm{H}_{3} \mathrm{~S}-\mathrm{R} 3 m-150 \\
\mathrm{GPa}\end{array}$ & \multicolumn{3}{c}{$\left|\mathrm{C}_{\mathrm{Q}}\right|(\mathrm{MHz})$} & \multicolumn{3}{c}{$\eta_{Q}$} \\
\hline Atomic site $\times \mathrm{N}$ & $\mathrm{PBE}$ & $\mathrm{PBE}+\mathrm{TS}$ & HSE & PBE & PBE+TS & HSE \\
${ }^{2} \mathrm{H} \times 6$ & $6.84 \mathrm{E}-02$ & $6.69 \mathrm{E}-02$ & $6.78 \mathrm{E}-02$ & 0.00 & 0.00 & 0.01 \\
${ }^{33} \mathrm{~S} \times 2$ & $7.40 \mathrm{E}-01$ & $1.18 \mathrm{E}+00$ & $4.96 \mathrm{E}-01$ & 0.00 & 0.00 & 0.00 \\
\hline
\end{tabular}




\section{Background theory of NMR spectroscopy}

(a)

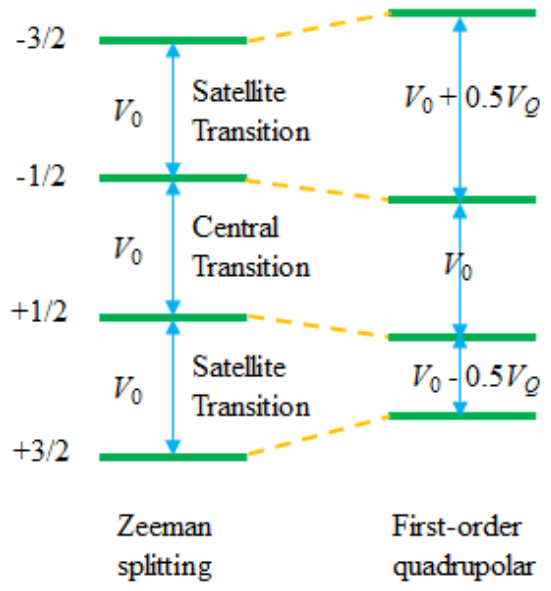

(c)

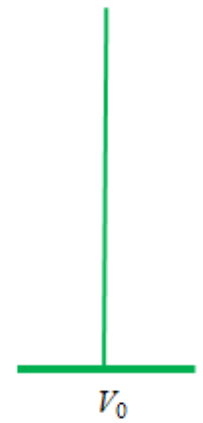

Zeeman splitting (d)

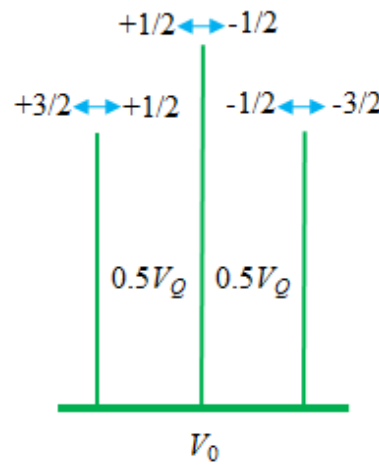

First-order quadrupolar

Figure S1. Schematic diagrams of the energy levels and corresponding NMR absorption spectra for the spin $I=3 / 2$ nucleus under the Zeeman and first-order quadrupolar interaction.

Figure S1 depicts the schematic diagrams of the energy-level splittings and corresponding NMR spectra under the quadrupolar interaction. For a spin $I=3 / 2$ nucleus, the degenerate energy level will split into $4(2 I+1)$ energy levels under the action of a magnetic field (the Zeeman splitting). Each energy level is equally spaced, and all of the energy-level transitions have frequency $V_{0}$. Hence, there is only one resonance line on the NMR spectrum. However, in general, the charges around the nucleus are not evenly distributed, and hence form an electric field that interacts with the quadrupolar moment of the nucleus, inducing the quadrupolar interaction which can be treated as a first-order perturbation to the Zeeman splitting in most cases. The firstorder quadrupolar interaction will make the energy-level intervals unequal and the energy levels arrange in an arithmetic sequence. The corresponding frequencies of the 
absorbed electromagnetic waves are $V_{0}-0.5 V_{Q}, V_{0}$ and $V_{0}+0.5 V_{Q}$, respectively. $V_{Q}$ is the quadrupolar splitting parameter and is given by:

$$
V_{Q}=V_{Q}^{\mathrm{PAS}}\left(1-\eta_{Q}\right)
$$

$V_{Q}^{\mathrm{PAS}}$ is the quadrupolar frequency (PAS denotes the principal axis system of the quadrupolar interaction) with the equation:

$$
V_{Q}^{\mathrm{PAS}}=3 C_{Q} /(2 I(2 I-1))
$$

Overall, there are three resonance lines on the NMR spectrum: one high spectral line corresponding to the central transition (CT) whose frequency of the absorbed wave is unaffected, and two low spectral lines corresponding to the satellite transition (ST) whose frequencies of the absorbed waves change.

\section{The quadrupolar asymmetry parameter of $\mathrm{H}_{3} \mathrm{~S}$ as a function of pressure}

Table S3. ${ }^{2} \mathrm{H}$ and ${ }^{33} \mathrm{~S} \eta_{\mathrm{Q}}$ values of different phases for $\mathrm{H}_{3} \mathrm{~S}$ as a function of pressure.

\begin{tabular}{cccc}
\hline $\mathrm{H}_{3} \mathrm{~S}$ & \multicolumn{3}{c}{$\eta_{\mathrm{Q}}$} \\
\hline Pressure (GPa) & 100 & 125 & 150 \\
${ }^{2} \mathrm{H}(\mathrm{Im}-3 m)$ & 0.00 & 0.00 & 0.00 \\
${ }^{33} \mathrm{~S}(\mathrm{Im}-3 m)$ & 0.00 & 0.00 & 0.00 \\
${ }^{2} \mathrm{H}(R 3 m)$ & 0.01 & 0.00 & 0.00 \\
${ }^{33} \mathrm{~S}(R 3 m)$ & 0.00 & 0.00 & 0.00 \\
\hline
\end{tabular}




\section{The EFG tensor parameters of LaH10 structures}

Table S4. The EFG tensor parameters of the Fm-3m phase for $\mathrm{LaH}_{10}$.

\begin{tabular}{ccccccc}
\hline $\mathrm{LaH}_{10}-\mathrm{Fm}-3 m$ & \multicolumn{3}{c}{$\left|C_{Q}\right|(\mathrm{MHz})$} & \multicolumn{3}{c}{$\eta_{Q}$} \\
\hline Atomic site $\times \mathrm{N}$ & $150 \mathrm{GPa}$ & $175 \mathrm{GPa}$ & $200 \mathrm{GPa}$ & $150 \mathrm{GPa}$ & $175 \mathrm{GPa}$ & $200 \mathrm{GPa}$ \\
${ }^{2} \mathrm{H} \times 32$ & $4.23 \mathrm{E}-03$ & $4.57 \mathrm{E}-03$ & $4.84 \mathrm{E}-03$ & 0.00 & 0.00 & 0.00 \\
${ }^{2} \mathrm{H} \times 8$ & $7.69 \mathrm{E}-15$ & $4.81 \mathrm{E}-15$ & $1.05 \mathrm{E}-15$ & 0.00 & 0.00 & 0.00 \\
${ }^{139} \mathrm{La} \times 4$ & $2.47 \mathrm{E}-13$ & $5.69 \mathrm{E}-13$ & $3.10 \mathrm{E}-13$ & 0.00 & 0.00 & 0.00 \\
\hline
\end{tabular}

Table S5. The EFG tensor parameters of the $R-3 m$ phase for $\mathrm{LaH}_{10}$.

\begin{tabular}{ccccccc}
\hline $\mathrm{LaH}_{10}-\mathrm{R}-3 \mathrm{~m}$ & \multicolumn{3}{c}{$\left|C_{Q}\right|(\mathrm{MHz})$} & \multicolumn{3}{c}{$\eta_{\mathrm{Q}}$} \\
\hline Atomic site $\times \mathrm{N}$ & $150 \mathrm{GPa}$ & $175 \mathrm{GPa}$ & $200 \mathrm{GPa}$ & $150 \mathrm{GPa}$ & $175 \mathrm{GPa}$ & $200 \mathrm{GPa}$ \\
${ }^{2} \mathrm{H} \times 18$ & $3.49 \mathrm{E}-03$ & $3.85 \mathrm{E}-03$ & $4.16 \mathrm{E}-03$ & 0.09 & 0.01 & 0.03 \\
${ }^{2} \mathrm{H} \times 6$ & $7.02 \mathrm{E}-03$ & $6.29 \mathrm{E}-03$ & $6.03 \mathrm{E}-03$ & 0.00 & 0.00 & 0.00 \\
${ }^{2} \mathrm{H} \times 6$ & $1.24 \mathrm{E}-03$ & $6.59 \mathrm{E}-04$ & $3.92 \mathrm{E}-04$ & 0.00 & 0.00 & 0.00 \\
${ }^{139} \mathrm{La} \times 3$ & $1.30 \mathrm{E}+00$ & $1.46 \mathrm{E}+00$ & $1.49 \mathrm{E}+00$ & 0.00 & 0.00 & 0.00 \\
\hline
\end{tabular}

Table S6. The EFG tensor parameters of the $C 2 / m$ phase for $\mathrm{LaH}_{10}$.

\begin{tabular}{ccccccc}
\hline $\mathrm{LaH}_{10}-\mathrm{C} 2 / m$ & \multicolumn{3}{c}{$\left|C_{Q}\right|(\mathrm{MHz})$} & \multicolumn{3}{c}{$\eta_{Q}$} \\
\hline Atomic site $\times \mathrm{N}$ & $150 \mathrm{GPa}$ & $175 \mathrm{GPa}$ & $200 \mathrm{GPa}$ & $150 \mathrm{GPa}$ & $175 \mathrm{GPa}$ & $200 \mathrm{GPa}$ \\
${ }^{2} \mathrm{H} \times 8$ & $2.92 \mathrm{E}-02$ & $2.89 \mathrm{E}-02$ & $2.75 \mathrm{E}-02$ & 0.87 & 0.85 & 0.84 \\
${ }^{2} \mathrm{H} \times 8$ & $3.08 \mathrm{E}-02$ & $1.54 \mathrm{E}-02$ & $1.12 \mathrm{E}-02$ & 0.77 & 0.64 & 0.40 \\
${ }^{2} \mathrm{H} \times 4$ & $1.67 \mathrm{E}-02$ & $1.34 \mathrm{E}-02$ & $1.27 \mathrm{E}-02$ & 0.63 & 0.61 & 0.54 \\
${ }^{2} \mathrm{H} \times 4$ & $2.51 \mathrm{E}-02$ & $1.70 \mathrm{E}-02$ & $1.49 \mathrm{E}-02$ & 0.08 & 0.00 & 0.08 \\
${ }^{2} \mathrm{H} \times 4$ & $1.72 \mathrm{E}-02$ & $1.60 \mathrm{E}-02$ & $1.52 \mathrm{E}-02$ & 0.10 & 0.07 & 0.27 \\
${ }^{2} \mathrm{H} \times 4$ & $1.65 \mathrm{E}-02$ & $1.21 \mathrm{E}-02$ & $1.22 \mathrm{E}-02$ & 0.47 & 0.37 & 0.22 \\
${ }^{2} \mathrm{H} \times 4$ & $1.23 \mathrm{E}-02$ & $1.20 \mathrm{E}-02$ & $1.05 \mathrm{E}-02$ & 0.91 & 0.70 & 0.53 \\
${ }^{2} \mathrm{H} \times 4$ & $1.03 \mathrm{E}-02$ & $9.67 \mathrm{E}-03$ & $9.24 \mathrm{E}-03$ & 0.80 & 0.94 & 0.95 \\
${ }^{139} \mathrm{La} \times 4$ & $6.83 \mathrm{E}+00$ & $5.23 \mathrm{E}+00$ & $4.69 \mathrm{E}+00$ & 0.86 & 0.68 & 0.62 \\
\hline
\end{tabular}

\section{REFERENCES}

1. Clark, S. J.; Segall, M. D.; Pickard, C. J.; Hasnip, P. J.; Probert, M. I. J.; Refson, 
K.; Payne, M. C., First principles methods using CASTEP. Z. Kristallogr. 2005, 220, 567-570.

2. Perdew, J. P.; Burke, K.; Ernzerhof, M., Generalized gradient approximation made simple. Phys. Rev. Lett. 1996, 77, 3865.

3. Tkatchenko, A.; Scheffler, M., Accurate molecular van der Waals interactions from ground-state electron density and free-atom reference data. Phys. Rev. Lett. 2009, 102, 073005.

4. Krukau, A. V.; Vydrov, O. A.; Izmaylov, A. F.; Scuseria, G. E., Influence of the exchange screening parameter on the performance of screened hybrid functionals. $J$. Chem. Phys. 2006, 125, 224106.

5. Monkhorst, H. J.; Pack, J. D., Special points for Brillouin-zone integrations. Phys. Rev. B 1976, 13, 5188.

6. Bonhomme, C.; Gervais, C.; Babonneau, F.; Coelho, C.; Pourpoint, F.; Azais, T.; Ashbrook, S. E.; Griffin, J. M.; Yates, J. R.; Mauri, F., First-principles calculation of NMR parameters using the gauge including projector augmented wave method: a chemist's point of view. Chem. Rev. 2012, 112, 5733.

7. Pyykko, P., Year-2008 nuclear quadrupole moments. Mol. Phys. 2008, 106, 19651974.

8. Monserrat, B.; Ashbrook, S. E.; Pickard, C. J., Nuclear magnetic resonance spectroscopy as a dynamical structural probe of hydrogen under high pressure. Phys. Rev. Lett. 2019, 122, 135501. 